\title{
Cocaínas fumables en Latinoamérica
}

\author{
Castaño, G.A. \\ Médico, "Magister" en Drogodependencias. Coordinador Nacional Post-grado Especialización en Farmacodependencia. FUNLAM. Medellín. Colombia.

\section{RESUMEN}

La cocaína es frecuentemente fumada en el continente americano en aquellas formas que permiten hacerlo: " el Basuco," "el Crack" y "la Base Libre." Las "cocaínas fumables" derivan del procesamiento de la hoja de coca y tienen un muy bajo punto de fusión, lo que les permite ser volatilizadas fácilmente lo que les da un mayor poder adictivo, produciendo en los consumidores una rápida dependencia y un grave deterioro biopsicosocial.

El consumo de pasta básica de cocaína aparece circunscrita a principios de la década de los '70 al área Andina en países como Perú, Colombia, Bolivia y Ecuador, casi al mismo tiempo que se reportan los primeros casos de uso de base libre en los Estados Unidos de América. Los índices de prevalencia de vida elaborados a partir de diversos estudios son: Colombia 1.5\%; Perú $3.1 \%$; Bolivia $1.7 \%$ y Ecuador $1.0 \%$, con un predominio de consumo en el sexo masculino.

En general los planteamientos propuestos para el tratamiento de adictos a las "cocaínas fumables", han sido diversos y en ocasiones controvertidos. Los resultados hasta ahora no son satisfactorios, la retención en los procesos de rehabilitación han sido pobres y las recaídas frecuentes, lo que ha hecho que algunos expertos incluyan dentro del arsenal terapéutico la aplicación de estrategias de Reducción de Daño. Algunas experiencias en este sentido han sido desarrolladas en países como Colombia, Bolivia y Brasil.

Palabras Claves: Cocaínas fumables, "basuco," "crack," "base libre," Latinoamérica, epidemiología, clínica, terapéutica.

\section{SUMMARY}

Cocaine is frequently smoked on the American continent in such forms as basuco, crack, and freebase. The smokable cocaines are derivatives of the coca leaf and have a very low fusion point that makes them more volatile which gives them a greater addictive power, leading to rapid dependency and a serious biophysical deterioration.

At the beginning of the seventies, use of the basic coca paste appeared limited to the Andes area in such countries as Peru, Colombia, Bolivia and Ecuador, almost at the same time that the first cases of freebase use were being reported in the United States. The life prevalence indices elaborated on the basis of these studies are: Colombia 1.5\%, Peru 3.1\%; Bolivia $1.7 \%$ and Ecuador $1.0 \%$ with a predominance of use among men.

In general, there have been many - and, on occasion, controversial-treatment proposals for smokable cocaine addicts. Results up to now have not been satisfactory. Rates for remaining in rehabilitation have been poor and relapses frequent. This has led to some experts including the application of harm reduction strategies in the therapeutic arsenal. Some experiences of this nature have been developed in such countries as Colombia, Bolivia and Brazil.

Key words: smokable cocaine, basuco, crack, freebase, Latinamerica, epidemiology, clinic, therapy.

\section{INTRODUCCIÓN}

D urante los últimos doce siglos, en los cuales al menos se ha tenido conocimiento de la planta de la coca y ciento cincuenta años después desde que Nieman aisló por primera vez la sustancia que denominó Cocaína, ésta ha representado un papel importante en muchos momentos de la vida de la humanidad.
Fue droga de altas jerarquías sacerdotales, de aristócratas, de científicos y de muchas personalidades sobresalientes en diferentes épocas. Existe voluminosa documentación a nivel antropológico donde se expone el uso médico y mágico de la planta de la coca, en la época preincaica, y luego en la civilización Inca.

Uno de los hitos mas destacados de la historia de ésta planta fue la obra Travels in Perú (Viajes por Perú) 
del eminente naturalista suizo J.J. Von Tshudi' ${ }^{1}$, obra escrita en 1847, texto de necesaria consulta cuando se quiere conocer los inicios de consumo de la coca.

Atacado y condenado su consumo por los aborígenes americanos en la primera Asamblea Eclesiástica celebrada en Lima en el año de 1551, por considerarse unida a la idolatría y a la hechicería; es alabada y recomendada por su principio activo - la cocaína - y sus acciones farmacológicas a finales del siglo XIX, cuando Sigmun Freud y Halsted y Hall, lo utilizaron en sus experimentos de psicofarmacología (1) y de anestesia local, respectivamente.

Muchos han sido los estudios realizados a esta sustancia, desde la óptica de sus efectos psicoactivos; desde los realizados por Freud en 1894 con su obra Über Coca, y los del farmacólogo Francis Louis Levin en su obra Phantastica publicada en 1924, hasta los mas recientes y que tienen que ver con los mecanismos moleculares de la adicción a la cocaína publicados por Leshner Al, en el New England Journal Medical del año de 1996 (2), las investigaciones para el desarrollo de la Vacuna contra la cocaína (3) y el ensayo de nuevos medicamentos como el Vigavatrin(gammavinil GABA- GVG) en el tratamiento de los adictos (4). Sin embargo, todavía poco se conoce acerca de algunos de algunos de sus derivados: el Crack, la base libre y el basuco (pasta básica), formas fumables de la cocaína.

\section{COCAÍNAS DE COMBUSTIÓN}

Se denominan así aquellas sustancias derivadas de la hoja de coca, que después de su procesamiento químico, adquieren características fisico-químicas que le dan puntos de fusión bajos y que pueden ser volatilizadas por sublimación o ebullición, utilizando calor.

Los alcaloides son sustancias químicas capaces de formar sales en presencia de ácidos. En general el término alcaloide es muy poco preciso y se aplica a un grupo heterogéneo de sustancias nitrogenadas extraídas de vegetales y que tienen acción farmacológica. La mayoría son sustancias básicas, aunque algunas como la colchisina son neutras.

La cocaína se define como un alcaloide y tiene las características clásicas de éstos: base nitrogenada capaz de formar sales con ácidos orgánicos e inorgánicos. Origen vegetal y actividad farmacológica definida.

A las cocaínas fumables ("basuco," "crack" y "base libre"), se les define como sustancias volátiles y sublimables, es decir que pueden pasar a fase gaseosa directamente desde lo sólido. Son líquidas desde los
98 grados centigrados y su punto de ebullición esta entre los 187 y 188 grados centígrados (5). La sal clorhidrato de cocaína, tiene un punto de fusión muy alto, lo que impide su sublimación, por lo tanto no se puede fumar, pues es destruida por el calor.

\section{Base libre y Crack.}

Ambas son dos formas base de las cocaínas fumables, químicamente iguales entre ellas, que difieren fundamentalmente por su proceso de elaboración. Ambas se obtienen a partir del clorhidrato de cocaína mediante procedimientos empíricos y rudimentarios de laboratorio que los mismos consumidores incluso pueden realizar y al cual ellos mismos vulgarmente llaman "patraseo" (volver atrás). Diagrama 1.

\section{PROCESO SIMPLIFICADO DE PRODUCCIÓN DE LAS COCAÍNAS FUMABLES}

\section{HOJAS DE COCA}

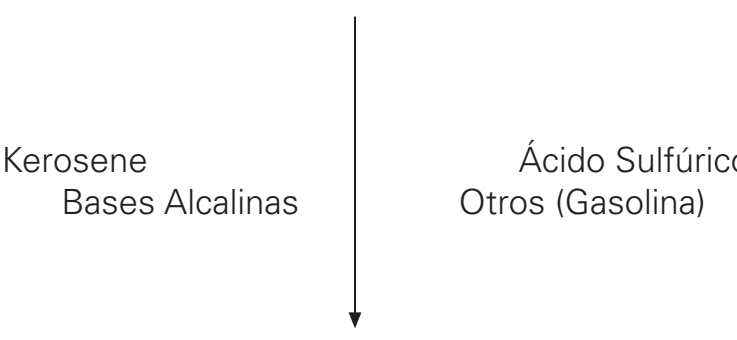

Pasta de Coca (Basuco)

(Fumable)
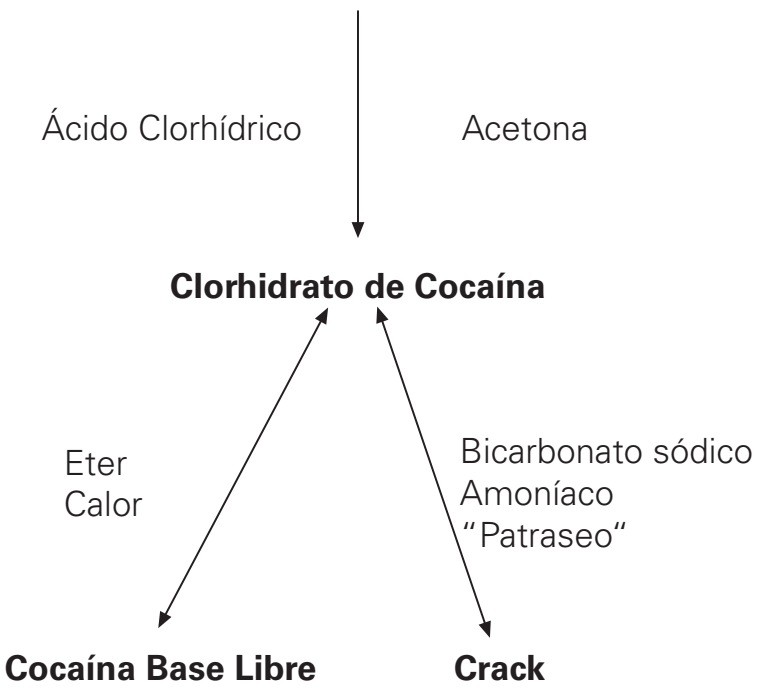

(Fumable)

(Fumable)

Diagrama $\mathbf{n}^{\circ}$ 1. Este diagrama muestra esquemáticamente como se procesan las diferentes formas de cocaínas fumables. El último paso para la obtención de "Base Libre" y "Crack" es lo que vulgarmente se denomina "patraseo". 
La base libre se obtiene añadiendo éter y calor elevado al clorhidrato de cocaína. En cambio si lo que se utiliza es bicarbonato sódico, amoniaco y agua con calor moderado, el producto final será el Crack.

La producción de Base libre se ha restringido a aquellos consumidores que quieren producir cantidades pequeñas para su propio uso, pues su producción industrial resulta peligrosa por la manipulación del éter que es un disolvente altamente inflamable lo que conlleva al riesgo de incendios y explosiones. La apariencia de la base libre es un polvo blanco y su pureza puede alcanzar el 95\% o más. La Base libre se fuma en "pipas de agua", las cuales pueden ser construidas a partir de latas de refresco, botellas de plástico o cristal, tubos de cristal, vasos y papel de aluminio. La droga se calienta cuidadosamente en la pipa hasta que se vaporiza y se pueden inhalar los gases, proceso que se conoce como "freebasing".

Los peligros en la producción de la Base libre y lo engorroso de su consumo, dio lugar al surgimiento del Crack, "rock" o "piedra", nombres vulgares con los que también se conoce. El Crack, una vez seco, tiene forma de porcelana que se tritura en escamas gruesas. Se fuma en recipientes en forma de pipa donde se pulveriza y se fuma. También puede mezclarse con tabaco y marihuana, en forma de cigarrillos, aunque los consumidores prefieren el consumo en pipa. El nombre de Crack procede del ruido de crepitación que producen los cristales cuando de calientan, presuntamente causados por el bicarbonato de sodio. Su pure- za puede alcanzar el 100\%. El consumo de crack esta muy difundido en los E.E.U.U. de Norteamérica.

\section{Pasta Básica de Cocaína.}

También se denomina sulfato de Cocaína, pasta base o simplemente pasta y con el nombre vulgar de Basuco y/o Bazuca en Colombia, Pitillo en Bolivia, Baserolo en Ecuador y Pasta de Coca en el Perú; es un producto intermedio en la fabricación de clorhidrato de cocaína a partir de las hojas de coca. Generalmente se obtiene al disolver en agua las hojas de coca secas y tratar la solución con Kerosene o gasolina, para posteriormente hacerlo con sustancias alcalinas y permanganato de potasio y finalmente con ácido sulfúrico.

La pasta básica - Basuco- producida en Colombia es diferente a la descripción tradicional que se ha hecho de que es un producto intermedio en la preparación del Clorhidrato de Cocaína. En Colombia el "Basuco" es un remanente en el segundo paso de pasta de coca a cocaína base, obtenido por métodos sencillos, utilizando Kerosene, sustancias básicas, ácido sulfúrico y éter (6). Diagrama 2. Producción del Clorhidrato de Cocaína.

Puede encontrarse en el mercado como pasta blanca lavada o pasta parda bruta, la cual es más barata.

El producto que se obtiene es un polvo blanco amarillento, untuoso, de consistencia pastosa y de un

\section{PRODUCCIÓN DE CLORHIDRATO DE COCAÍNA}

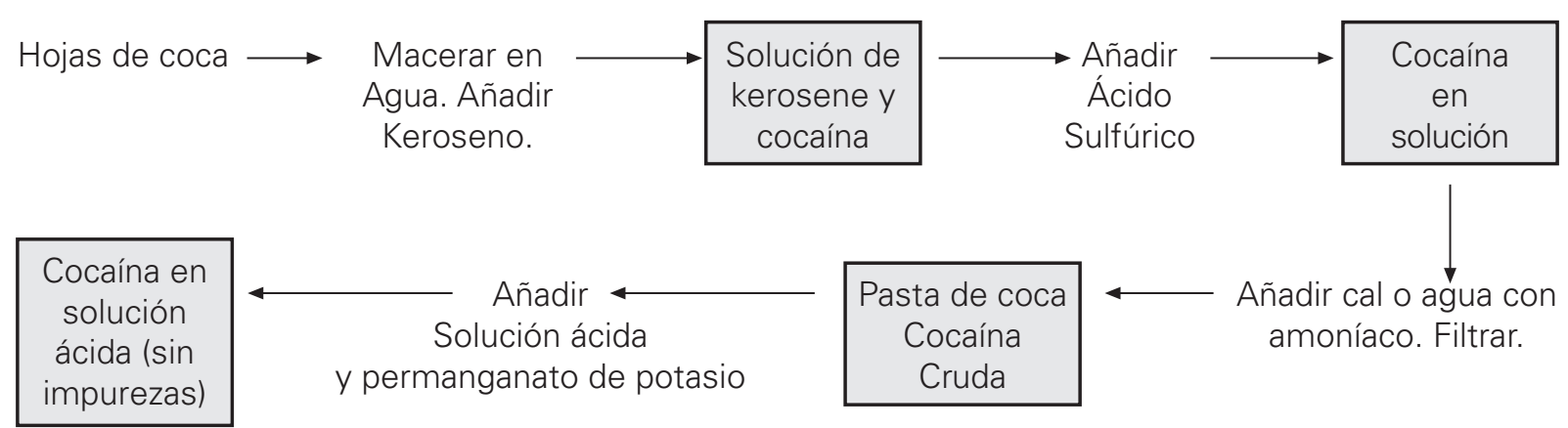<smiles>[13CH3]</smiles>

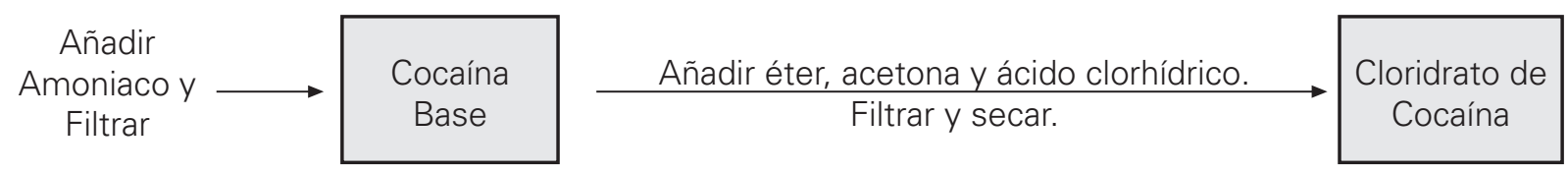

Diagrama $\mathbf{n}^{\circ}$ 2. El diagrama de la producción muestra como se procesan las hojas de coca hasta llegar al clorhidrato de cocaína. Normalmente se hace en Sudamérica, antes de exportar la droga, pero a veces se exportan tanto las hojas como la pasta de coca para su refinamiento en otro lugar. 
olor muy penetrante. Contiene entre un $40 \%$ a un $85 \%$ de sulfato de cocaína y sirve para la posterior elaboración del clorhidrato de cocaína. Se consume fumada en pipa o mezclado con tabaco o marihuana en cigarrillo.

Aparte de las impurezas de origen ( contaminación con productos derivados del petróleo, carbonatos (cemento), etc.), se le adultera con harina de trigo, polvo de ladrillo y azúcar morena, procedimiento realizado por los pequeños traficantes para hacerla rendir y obtener mayores ganancias.

El Basuco generalmente se consume fumado, armado en cigarrillos con filtro, a los cuales les extraen más o menos la mitad de la picadura (tabaco), para completarlo con el polvo del basuco. El filtro se parte por la mitad para lograr una mayor aspiración. También lo consumen mezclado con marihuana para disminuir los efectos "angustiantes" y "paranoides" de la pasta básica. A esta mezcla la denominan "banano", "maduro" o "pecoso", pues la marihuana al calentarse produce en el papel unas manchas en forma de "pecas", lo que le da la apariencia de un banano (plátano) pecoso.

Otra forma de consumo, muy extendida en Colombia, es su consumo en pipa, la cual es construida artesanalmente, por los mismos consumidores utilizando pequeñas botellas de plástico, lata o vidrio, a las que le adaptan la "coca" vacía de un bolígrafo. La parte superior del recipiente es cubierta con papel de aluminio, al cual le hacen pequeños agujeros. Sobre esta superficie vierten ceniza "pavesa" de cigarrillo, a la cual le agregan el polvo untuoso del basuco, luego es calentado con un mechero, produciéndose los vapores que son aspirados por el consumidor.

La pasta básica de cocaína tiene bajo costo y una alta disponibilidad en países del Área Andina Latinoamericana, pues se obtiene mediante procesos de laboratorio muy simples, es de fácil uso y posee una potente y breve acción, lo que la hace muy adictiva.

\section{PREVALENCIA DE CONSUMO DE LAS COCAÍNAS FUMABLES.}

Sólo hasta comienzos del siglo XX la preocupación por casos de adicción a la cocaína se hizo evidente y es cuando de manera sistemática se empieza a controlar el cultivo de la hoja de coca.

El consumo de ésta sustancia aspirada por la nariz, se extiende por E.E. U.U. de Norteamérica, al tiempo que en Europa su consumo se circunscribe a ambientes artísticos y bohemios de las grandes ciudades, aunque éste se aumenta considerablemente en muchos países de Europa y especialmente en Francia, durante y después de la Segunda Guerra Mundial.
A partir de los años 30, el consumo global de clorhidrato de cocaína disminuye sustancialmente, coincidiendo con la aparición de un boohm en el consumo de heroína.(7) Desde entonces, la historia en las prevalencias de su consumo ha sufrido altibajos: en 1954, la comisión de expertos de la ONU en Farmacodependencias observó "con satisfacción la disminución y escasa calidad de las incautaciones de cocaína" (7).

En la década de los '70, el consumo de clorhidrato de cocaína tiene un repunte importante y desde mediados de los '80 la adicción a ésta sustancia constituye uno de los problemas mas graves del mundo occidental.

La primera revisión bibliográfica hecha sobre la cocaína fumada fue realizada por Siegel en 1982 (8), donde señalaba que el primer reporte sobre el empleo de cigarrillos de hojas de coca, había sido hecho por Stewart en 1885, con efectos muy pobre sobre el SNC.

El consumo de pasta básica de cocaína aparece circunscrita a principios de la década de los '70 al área Andina en países como Perú, Colombia, Bolivia y Ecuador. Casi al mismo tiempo se reportan los primeros casos de uso de base libre en los Estados Unidos de América (9)

La primera descripción de un caso clínico sobre el consumo de pasta básica de cocaína que se conoce, aparece registrado un día de invierno de 1972, en el Hospital Hermilio Valdizan en Lima, Perú. Ese día el Dr. Humberto Rotondo se muestra sorprendido por las características peculiares y sintomatología que presenta un joven, que manifestó consumir cocaína fumada; "pasta base" la llamaba él. Rafael Navarro Cueva del Centro de Rehabilitación de Farmacodependientes de Naña, del mismo Hospital y colega del Dr. Rotondo, relata así estas primera experiencias: “ Nuestras primeras observaciones advertían experiencias de euforia y placer mínimas durante la intoxicación; mas bien el paciente decía "no comprender porque consumía la pasta básica"...... En el periodo de intoxicación predominaba la angustia y sobre todo una compulsión indescriptible por seguir consumiendo. También nos percatamos de una devastación cada vez más intensa de los hábitos, de la alimentación y cuidado personal;.... Todo esto nos llevo a la conclusión de que estabamos frente a un nuevo, interesante y grave desorden psicopatológico" (9).

Mas tarde en 1976, Alameida (10), Ilama la atención sobre la dependencia a la pasta básica asociada con el uso de alcohol y simultáneamente Jeri, Sanchez y del Pozo(11), identifican a siete pacientes con problemas de uso de pasta básica, dentro de un grupo de 79 usuarios de drogas pertenecientes a las fuerzas armadas y fuerzas policiales ( o parientes de ese personal) en el Perú. 
El número de pacientes se fue incrementando, sucesiva y gradualmente, al punto que los interesados en Salud Pública del Perú, señalaban el carácter epidémico del consumo, aludiendo al poder adictivo de la sustancia, la contagiosidad social de su uso y la rapidez y amplitud de su esparcimiento (9).

Los estudios más juiciosos y sistemáticos sobre el consumo de la pasta básica de cocaína, han sido realizados en el Perú; son de recalcar los trabajos de $\mathrm{M}$. Fernández G. (12, 13, 14); E. Sánchez (15, 16, 17); R. Navarro y Colb (18); E Oliber y Colb (19, 20, 21); M. Almeida (22); C. Carbajal Y Colb (23); F.R. Jerry y Colb $(24,25,26,27)$, y M. Nizama (28). En ellos se hacen contribuciones importantes en aspectos clínicos, morbilidad hospitalaria y tratamiento. En dos de ellos (19 y 20), se investiga prevalencia de uso en estudiantes de enseñanza secundaria y el (23), prevalencia de consumo en la población general de Lima, Metropolitana.

En el resto de países en donde esta forma de cocaína es consumida; Colombia, Bolivia y Ecuador: los estudios realizados, han sido mas de corte epidemiológico.

En Colombia el consumo de Basuco se inicio en forma epidémica aproximadamente en 1983, posiblemente como resultado de un subproducto del procesamiento y tráfico de la cocaína.

En este país, el último de los estudios realizado en una muestra de 18.770 personas, con rangos de edad entre los 12 y los 60 años, por la Dirección Nacional de Estupefacientes (29), organismo gubernamental que lleva las políticas de drogas en el país, reportó un consumo de basuco al menos una vez en la vida de 385.626 consumidores $(1,5 \%)$; un porcentaje muy similar al consumo de cocaína (1,6\%). Entre los consumidores predomina el sexo masculino $(2,5 \%)$, frente al femenino $(0,6 \%)$, siendo unos 282.000 consumidores hombres y 61.000 mujeres.

La prevalencia por grupo de edad muestra que el grupo de mayor consumo es el de los 25 a 44 años $(2,6 \%)$. Con respecto al nivel de educación la mayoría de los consumidores de basuco en Colombia han alcanzado un nivel universitario $(2,6 \%)$, seguida de educación secundaria completa $(1,6 \%)$, incompleta $(1,5 \%)$, primaria completa $(1,4 \%)$ e incompleta $(1,2 \%)$ y sin educación $(0,7 \%)$.

Al igual que en el consumo de otras sustancias ilegales la prevalencia de vida mas alta de consumo esta en las personas desempleadas (7,6\%).

La edad promedio de inicio en el consumo, 18,2 años es la menor comparada con las otras sustancias psicoactivas. El consumo se hace con mayor frecuencia en zonas recreativas (parques), (71,6\%).

Casi el 50\% consume 1 a 3 veces al mes, mientras que el $0.9 \%$ lo hace todos los días y el 39,1\% nunca consume estando solo.
Los consumidores de Basuco de este estudio, reportaron haber tenido una mayor proporción de problemas que los consumidores de otras sustancias, principalmente problemas en el trabajo $(54,6 \%)$, económicos $(53,9 \%)$ y con la familia $(39,7 \%)$.

Datos más recientes sobre el consumo de basuco en Colombia, están recogidos en el VESPA Universitario de la Cuidad de Medellín (30). Este estudio tomó como muestra 3.749 estudiantes, de todos los semestres, de programas de pregrado en las modalidades diurna y nocturna de 18 universidades de la ciudad de Medellín y Area Metropolitana. La proporción de prevalencia de consumo de basuco en la vida reportada por el estudio fue: prevalencia de vida (16 por mil) y prevalencia anual ( 9 por mil), presentándose una mayor frecuencia de consumo en el sexo masculino (39 por mil).

El estudio trató de establecer también el consumo de marihuana como factor de riesgo al consumo de basuco. Los resultados arrojados dieron cuenta que: el consumo de basuco en los consumidores de marihuana, presentó una razón de 11.99, con intervalos de confianza del $95 \%$ entre $5.56<R$ D $<26.07$ y una significancia estadística muy fuerte de un valor de $P=0.000000$, confirmando el comportamiento del consumo de marihuana como un factor de riesgo para el consumo de basuco.

En el Perú, uno de los más recientes estudios fue realizado por CEDRO: Rojas en 1995 (31). En una muestra de 6.379 sujetos, de ciudades con mas de 200.000 habitantes, cuyos rangos de edad estaban entre los 12 y los 50 años, se encontró una prevalencia de vida para el consumo de pasta básica del 3,10\% (193.550 personas), de los cuales el 0,7\% (23.281) eran mujeres y un 5,7\% eran hombres.

Según la "Segunda Encuesta Nacional sobre Consumo de Drogas en Ecuador - 1995 -CONSEP, SEVIP, NAS" (32), el consumo de drogas ilegales alguna vez en la vida, en la población entre 12 y 49 años fue de 347.592 ecuatorianos aproximadamente.

Entre los consumidores, la prevalencia de vida para el consumo de "baserolo" ( nombre vulgar con el que denominan a la pasta base), fue del 1,0\%.

En Bolivia, Alcaraz del Castillo F y otros (33), tomando como muestra 13.543 estudiantes de colegios públicos y privados, en sectores urbanos y rurales, con rangos de edad entre los 12 y los 21 años, encontraron prevalencias de vida para el consumo de pasta básica del $1,7 \%$, con una prevalencia en mujeres del 0,6\% y 2,6\% en varones (cuatro hombres por una mujer). El 1,2\% del total de estudiantes encuestados, había consumido en el último año, pasta base, una o más veces. La prevalencia de consumo en el último mes o consumo actual, encontrada fue del $0,6 \%$ y la edad de inicio al consumo promedio era de 14,4 años. 
Argentina presenta una prevalencia de vida para el consumo de Pasta base del 0,9\%, según el reporte de la Secretaria de Programación de la Drogadicción y la Lucha contra el Narcotráfico (34).

Chile en tasas globales, presenta tasas de prevalencia de vida para el consumo de pasta base del $1,9 \%$ y una incidencia (casos nuevos) del 0,5\%, según el Segundo Estudio Nacional sobre Consumo de Drogas realizado en ese país en 1996 (35).

El consumo de Crack, ha tenido un desarrollo importante, durante los últimos años en Brasil. Un informe sobre el consumo de esta sustancia es presentado por Bueno R y Carvalho en 1999 (36). En el estudio se reporta la substitución del consumo de cocaína intravenosa en varias ciudades del este país, por el uso de Crack fumado. En el se informa que más del $30 \%$ de los usuarios de cocaína inyectada, se están pasando al consumo de esta forma fumable de cocaína.

Informes anteriores de consumo de Crack en Brasil, fueron reportados por el Departamento de Investigación sobe Narcotráfico del Brasil- DENARC -. En el año 1996, el número de personas que fueron atendidas por dependencia a alguna sustancia psicoactiva fue de 915 . El 10,6\% fueron mujeres y el $84,9 \%$ hombres. La droga de elección entre los hombres era el Crack $(47 \%)$. Las mujeres que consumían crack, constituían el 36,1\% del total de mujeres de la muestra.(37).

Algunos estudios epidemiológicos realizados en Venezuela nos pueden dar idea del consumo de pasta básica en ese país. Una Encuesta Domiciliaria sobre Salud y Alcoholismo en la Población del Municipio de Baruta, en el Estado de Miranda, realizada entre 1.860 personas, por Simonovis N y otros en 1991 (38), reportó una prevalencia de vida para el consumo de pasta base del 0,5\%.

Otro estudio, donde la muestra estuvo constituida por el universo de los nuevos casos atendidos entre 1986 y 1994, en los Programas de Tratamiento de la Fundación José Felix Ribas de la ciudad de Maracaibo, Estado de Zulia (39). En un total de 853 sujetos reportó como la droga mas consumida para el momento de la entrevista, al basuco con el 36\%.

Finalmente y para dar una idea del consumo de esta sustancia en diferentes grupos poblacionales de Venezuela, me referiré a un estudio realizado por Salazar M y Ramos G, en 1994 (40). La muestra de esta investigación conformada por 2.014 personas entre estudiantes, docentes, empleados y obreros adscritos a una Universidad, reportó una prevalencia de vida para el consumo de basuco del 1,0\%.

Para culminar con esta mirada epidemiológica al consumo de cocaínas fumables, es importante anotar que el uso de base libre, sin conocerse estudios sistemáticos ha sido reportada en Perú, Ecuador y Chile, con una tímida extensión a Argentina (41).

\section{ASPECTOS CLÍNICO - FARMACOLÓGICOS Y TOXI- COLÓGICOS DE LAS COCAÍNAS FUMABLES.}

Las formas fumables de cocaína; basuco, base libre y crack, alcanzan rápidamente concentraciones plasmáticas del $70 \%$ al $80 \%$ tras su administración. Figura No 1.

\section{COCAÍNAS FUMABLES, GRADOS DE PUREZA, APARICIÓN DE EFECTOS Y NOMBRES VULGARES CON LOS CUALES SE CONOCEN.}

\begin{tabular}{|c|c|c|c|c|}
\hline PRODUCTO & $\begin{array}{l}\text { FORMA DE } \\
\text { CONSUMO }\end{array}$ & PUREZA & $\begin{array}{l}\text { APARICIÓN DE } \\
\text { EFECTOS }\end{array}$ & $\begin{array}{l}\text { NOMBRE } \\
\text { VULGAR }\end{array}$ \\
\hline Hojas de Coca & Masticada & $0.7 \%$ & $10-30$ minutos & \\
\hline Pasta Base & Fumada & $40-50 \%$ & 5 a 10 segundos & $\begin{array}{c}\text { Pasta base - Basuco, } \\
\text { Bazuca, Pitillo, } \\
\text { Baserolo }\end{array}$ \\
\hline Clorhidrato de Cocaína & $\begin{array}{l}\text { Esnifada, } \\
\text { inyectada }\end{array}$ & $25-100 \%$ & 30 a 50 segundos & $\begin{array}{c}\text { Cocaína - Nieve - } \\
\text { Coca - Perico }\end{array}$ \\
\hline Cocaína Base Libre & Fumada & $50-95 \%$ & 5 a 10 segundos & Base \\
\hline Crack & Fumada & $40-100 \%$ & 5 a 10 segundos & Rock - Roca - Piedra \\
\hline
\end{tabular}

Figura No. 1. La figura No. 1 describe esquemáticamente las formas de consumo, pureza, aparición de los efectos y nombres vulgares con los que más comúnmente se conocen las cocaínas fumables. 
Estas formas fumables de cocaína atraviesan rápidamente la barrera hematoencefálica tardando sólo cinco segundos en producir efectos centrales, a diferencia de los 30 segundos que tarda en aparecer los efectos en el SNC cuando es esnifado o aplicado por vía intravenosa, en forma de clorhidrato. La duración de sus efectos es también muy breve; tras su administración se produce un "flash" intenso y fugaz, desapareciendo rápidamente su acción, lo que sumerge al consumidor en una angustia, que lo obliga a ser compulsivo en el consumo. El metabolismo, mecanismos de acción y excreción del "basuco", "el crack" y la "base libre" son los mismos que se han descrito para el clorhidrato de cocaína.

Por ser similares los síntomas clínicos y toxicológicos de las dos formas de cocaínas fumables y por ser la sustancia sobre la cual tenemos más experiencia, nos referiremos sobre todo a la pasta básica de cocaína. Los síntomas clínicos - toxicológicos del consumidor de pasta básica de cocaína son sui géneris, pues difieren de los del usuario de hojas de coca en el coquismo y de los del usador de clorihidrato de cocaína en el cocainismo.

Nizama (28) y Navarro C. (42), en trabajos ya clásicos sobre el síndrome de la pasta básica de cocaína, realizaron extensas e interesantes descripciones de la fenomenología clínica y la historia natural de la dependencia a esta sustancia y que coincide con nuestra experiencia clínica en estos 10 años en el manejo de drogodependientes. El uso de pasta básica de cocaína por sus particularidades farmacológicas, produce desde su primer consumo un estado de intoxicación aguda, en la cual se pueden describir tres estadios clínicos, cada uno caracterizado por diversos síntomas. El primero denominado "momento antes del consumo", esta marcado por un síndrome obsesivo - compulsivo agudo, un síndrome ansioso y un síndrome neurovegetativo, caracterizado por sudoración, taquicardia y malestar abdominal referido como deseos de defecar. El segundo estadio "efectos al fumar", se inicia tras la primera aspirada y produce un "flash" placentero intenso (expansión placentera semejante al placer producido por el orgasmo), pero muy breve que dura apenas de 3 a 4 segundos, con una caída muy brusca. La angustia por la cesación de los efectos es tan fuerte que obliga al consumidor a fumar una y otra vez, a encender uno y otro cigarrillo, entrando en un estado de intensa angustia, irritabilidad e inquietud, cesando su consumo sólo cuando el dinero para su consumo se le ha terminado o cuando entra en un cuadro de intoxicación aguda.

Este cuadro esta caracterizado por los siguientes signos y síntomas:

Estimulación Vegetativa: tras las primeras fumadas se presentan signos y síntomas neurovegetativos que se van incrementando progresivamente como son el aumento de la frecuencia respiratoria y tensión arterial, taquicardia, hiperreflexia osteotendinosa, sudoración, parestesias, deseos de miccionar y de defecar, temblor en las extremidades, adormecimiento de los labios, náuseas y en algunos casos vómitos y sensación vertiginosa.

Hipersensibilidad sensorial: Al inicio parece haber aumento de sensibilidad olfatoria, incluso disfrutan del olor, aparece también una hipersensibilidad auditiva, que a medida que aumenta la intoxicación, se torna molesta.

Alteraciones psicomotrices: inicialmente hay incremento de la actividad motora. Cuando la intoxicación se hace muy manifiesta aparece una lentificación motora asociada con rigidez muscular especialmente en extremidades superiores y en mandíbula, así como la aparición de movimientos repetitivos de los brazos y manos, como si se estuviera tocando piano, y esterotipias motoras consistentes en movimientos de masticación y "chupeteo".

Síndrome Paranoide: Este síndrome se presenta en casi la totalidad de los consumidores de pasta básica de cocaína. Se inicia tempranamente pero alcanza su mayor intensidad al cabo del 5 cigarrillo. Los consumidores se tornan suspicaces, desconfiados, creen que son espiados o perseguidos, o que son descubiertos por una persona significativa. Los síntomas suelen desaparecer entre los 60 y 90 minutos después de terminar el consumo. Si los síntomas persisten seguramente se esta desarrollando un cuadro de psicosis paranoide.

Pseudo - percepciones: Las ilusiones son las primeras en presentarse, asociados al síndrome paranoide. El consumidor puede afirmar ver en otra persona o en un tronco, un policía y escuchar ruidos como pasos de alguien que lo espía. Posteriormente pueden aparecer verdaderas alucinaciones visuales y táctiles, sobre todo estas últimas, manifestadas como mircrozoopsias, pequeñas animales que andan por su cuerpo y producen picazón.

En el último de los estadios, definido por Nizama (28) como "post-efecto" y por Navarro(42) como "fase post-crítica", el consumidor estará fatigado, irritable, sudorosa y con deseos intensos de dormir. Al comienzo de la adicción pueden aparece intensos sentimientos de culpa y arrepentimiento por haber consumido, cosa que en el consumidor crónico no se presenta. Después de haber dormido, el usuario se mostrará agotado, con la boca seca y los labios y lengua enrojecidos e inflamados por el intenso consumo, así mismo se quejará de dolor faringeo, estará irritable y mostrará escasa tolerancia a la frustración y eventualmente cuadros depresivos mayores. Estos síntomas pueden desaparecer en pocas horas y dar paso a un nuevo episodio de consumo. 


\section{OTROS SIGNOS Y SÍNTOMAS DEL CONSUMO DE PASTA BÁSICA DE COCAÍNA}

La costumbre de fumar pasta básica de cocaína manteniendo entre los pulpejos del dedo índice y pulgar la sustancia, hace que se desarrolle una pigmentación de la piel de color amarillo ocre, resultado probablemente de las impurezas que acompañan a la cocaína base; este es un color más dorado que la pigmentación que deja la costumbre de fumar tabaco. Es frecuente también encontrar pigmentación similar a la de los pulpejos de los dedos en las encías y carrillos de los dientes centrales.

En los pulmones, el cuadro general es de una severa congestión pasiva aguda.

Son frecuentes los casos de enflaquecimiento, desnutrición y tuberculosis pulmonar en los consumidores crónicos de pasta básica de cocaína. La marginación social que padecen este tipo de consumidores en el Área Andina Latinoamericana es muy marcada; incluso los mismos consumidores de otras sustancias los discriminan.

El deterioro Bio-sico-social de los consumidores de pasta básica de cocaína es muy intenso, comparable a la que sufren los usadores de heroína y solo diferenciado por los problemas de orden biológico asociados al consumo de esta última sustancia por vía parental (SIDA, Hepatitis B y C, Septicemias, etc.).

\section{ESTRATEGIAS TERAPÉUTICAS EN LA REHABILITA- CIÓN DE ADICTOS A LA PASTA BÁSICA DE COCAÍ- NA}

Debido al carácter sui generis de la dependencia a la Pasta Básica de Cocaína, se ha generado un reto muy serio y muy difícil de resolver entre los terapeutas. El manejo de esta adicción presenta varios problemas complejos, la mayoría de los cuales todavía no se han resuelto de manera sistemática y científica, mediante estrategias adecuadas de investigación y continúa por tanto dependiendo de los profesionales expertos.

El tratamiento de adictos a Pasta Básica de Cocaína, comienza a llevarse a cabo durante los primeros años de la década del 70, época en que los tratamientos que se aplican son modalidades utilizadas para el tratamiento del alcoholismo y acomodados a las circunstancias y problemáticas concretas de este tipo de consumo (43). Los primeros tratamientos se basaban en las llamadas "curas de sueño", donde el paciente se le administraba medicamentos hipnóticos con el objeto de provocar un sueño profundo. Uno de los tratamientos más controvertidos, fue el utilizado en Perú, en el quinquenio 1980 - 1985: La Psicocirugía
(44). Esta consistía en un cingulotomía anterior bilateral $(C A B)$ y era realizada a adictos irrecuperables. En ella se removía tejido cerebral en una área del cortex denominada "girus cingulary", tratando de interferir reacciones bioquímicas, a través de la destrucción de receptores sinápticos. Las consecuencias nefastas en los usuarios de pasta básica de cocaína, a quien se les aplico, dan cuenta de las razones de su desuso.

Para disminuir la ansiedad "hambre de droga" se han intentado muchas y diversas terapias. Entre las farmacológicas con éxito limitado se han destacado el uso de la Bromocriptima y el Clorhidrato de Biperideno y la Buprenorfina; más recientemente se ha estado experimentando con el Flupentixol, un nuevo antagonista dopaminergico, el cual además de disminuir la ansiedad por el consumo (craving), producen un efecto antabuse en los consumidores (7).

En general los planteamientos terapéuticos que se propongan para el tratamiento de adictos a la Pasta Básica de Cocaína deben ser flexibles y personalizados, esto en razón de la existencia de diferentes escalas de dependencia que pueden presentar los usuarios y del mayor o menor deterioro bio-sico-social que presentan. Dentro de este amplio margen de posibilidades que pueden presentar los consumidores de Pasta Básica de Cocaína y utilizando el ASI (Índice de Severidad de la Adicción) y un inventario de redes sociales de apoyo se determina el tipo de intervención que el usuario necesita. En la gran mayoría de casos los consumidores de Pasta Básica de Cocaína son policonsumidores y cuando solicitan ayuda suelen tener ya un marcado deterioro bio-sico-social. Su retención en los tratamientos es pobre y las recaídas son frecuentes. Esto hace necesario que dentro del arsenal terapéutico que se utilice para la rehabilitación de estas personas se tengan en cuenta estrategias de reducción de daños.

En Colombia y más específicamente en Medellín, desde hace dos años, una Organización No Gubernamental viene desarrollando aplicando estrategias de Reducción de Daño en el tratamiento de consumidores de "basuco". Entre las más destacadas se encuentran: Programas de Tratamiento con Bajo Umbral de Exigencia, actividades con "educadores de calle" donde se educa en salud, se educa para la vida y se realizan acciones tendientes a cambiar las formas de consumo (cambiar el consumo de "basuco" en pipa y consumirla en cigarrillo, buscando con ello bajar la concentración de la sustancia pues el consumo en cigarrillo habitualmente se hace, mezclando la pasta básica con marihuana o con tabaco).

A los usuarios de este tipo de sustancia además de educarlos sobre los riesgos que plantea el consumo y motivarlos y sensibilizarlos para que inicien programas de rehabilitación, se les sugiere que en la mezcla, con marihuana o con tabaco, la cantidad de pasta básica de cocaína sea menor. 
Muchos esfuerzos se han llevado a cabo; la búsqueda de soluciones a ésta y otras formas de adicción seguirán siendo intensas; en los substitutos de cocaína, puede haber una esperanza alentadora.

Algunas experiencias sobre esta posible solución, han sido desarrolladas en Bolivia por el Dr. Jorge Hurtado(45), quien ha suministrado hojas de coca para "mascado" a dependientes de clorhidrato de cocaína y/o pasta básica que asisten a su consulta, y por Labigalini (46) en Brasil, quienes suministraron marihuana a abusadores de cocaína intravenosa y de crack, logrando resultados interesantes.

\section{BIBLIOGRAFÍA}

(1) QUEROL M,. Freud, la Coca y la Cocaína. En: Psicoactiva. Revista Científica. Centro de Información y Educación para la Prevención del Abuso de Drogas. 1996. Anexo X. No. 14; 81-101.

(2) LESHNER A, I. Molecular mechanisms of cocaina addiction. N. Engll. Med. 1996;335:128-129.

(3) CARRERA, M.R.A; ASHLEY, J.A.; PARSONS,L.H.; ET $A L$,. supression of psichoactive effects of cocaína by active inmunization. Nature, 1995. 378(14): 727-730.

(4) MARKOWITZ, J. S.; FINKEENBINE, R.; MYRICH, H.; ET $A L$, Gabapentin abuse in cocaina user: Implications for treatment . J. Clin. Psychofharmacol. 1997, 17: 423-424.

(5) SETCHER, P. G.; WINDHOLTZ, M.; LEAHY, D. S.; BOLTON, D. M. Y EATON, L. C. The Merck Index, 8va. Ed. 1968. Merck y Co. Rahway, New York. USA.

(6) CORDOBA PALACIO D.; TOLEDO ARENAS D,. Cocaína y base de Cocaína - "Basuco". En: Toxicología. Edit. Darío Córdoba. 3ra Edición. 1ra Reimpresión. Medellín, 1997. 645:313-320.

(7) LADERO P; LADERO J.M y OTROS. Drogodependencias: Farmacología, patología, Psicología, Legislación. Editorial Medica Panamericana. Madrid, 1999.

(8) SIEGELL, R. K.: Cocaina Smoking. Journal of Psychoactive Drugs, 1982.(14): 271-359.

(9) LEON F, R. Epidemiología del Uso y Abuso de la Pasta Básica de Cocaína en el Perú: 1976-1978, en: Pasta Básica de Cocaína, un estudio Multidisciplinario.Pps 29-111. CEDRO. Lima, 1989.

(10) ALAMEIDA, M. Alcohol y Drogas: Algunos aspectos de sus interrelaciones.. VI Congreso de Psiquiatría, Neurología y Neurocirugía, Lima, 1976.

(11) JERI, F, R.; SANCHEZ, C., y DEL POZO, T, Consumo de Drogas Peligrosas por Miembros y Familiares de la fuerza Armada y del Fuerza Policial
Peruana. Revista de la Sanidad del Ministerio del Interior, Lima, 1976. 37: 104-112.

(12) FERNÁNDEZ M, y COLABS. Balance y Perspectivas del Servicio de Alcoholismo y Farmacodependencia del Hospital "Víctor Larco Herrera". Primera Conferencia Internacional de Acción sobre el Abuso de Sustancias, Drogas y Alcohol. Phoenix, Atizona, USA. Nov. 1977.

(13) FERNÁNDEZ M.- El problema de las Drogas en el Perú. I Simposio Internacional de Alcoholismo y Farmacodependencia. Sao Paulo, Brasil. Nov. 1982

(14) FERNÁNDEZ M. - Aspectos Clínico-Sociales de la Drogodependencia (Perfil del Drogodependiente). Tesis Doctoral. Lima, 1984.

(15) SÁNCHEZ, E. Algunos Aspectos Epidemiológicos de la Dependencia a Pasta Básica de Cocaína. Rev. Neuropsiquiatría. Lima, 1979.

(16) SÁNCHEZ, E.- Estado Actual de la Farmacodependencia en el Perú. Rev. Peruana de Psiquiatría. "Hermilio Valdizan" Vol 1: 65 -73. Lima, 1963.

(17) SÁNCHEZ, E.- Sistemas de Tratamiento y Rehabilitación de Farmacodependientes. Análisis del Sexto Congreso Internacional de Psiquiatría "Hermilio Valdizan". Lima, 1980.

(18) NAVARRO, R y COLABS.- Terapia de la Conducta en el Comportamiento dependiente a Drogas. Tratamiento y Seguimiento de 26 casos clínicos. Rev. Peruana de Psiquiatría "Hermilio Valdizan" Vol I, 1: 24 - 30. Lima, 1983.

(19) OLIBER, E. y LLERENA, SH. Aproximación Discreta a la Epidemiología de la Farmacodependencia en Estudiantes Varones de Educación Secundaria Diurna de Lima. Metropolitana, abril 1979.

(20) OLIBER, E y CABANILLAS, L.- Algunos Aspectos Clínicos Observados en Consumidores de Pasta Básica de Cocaína. Lima, abril 1979.

(21) OLIBER, E.- Epidemiología de la Farmacodependencia en Estudiantes Secundarios de la Provincia Constitucional del Callao. Nov., 1981.

(22) ALAMEIDA, M.- Contribución al Estudio de la Historia Natural de la Dependencia a la Pasta Básica de Cocaína. Rev. Neuropsiquiatría. 41: 44 -45. Lima, 1978.

(23) CARBAJAL, C y JERE, F.R.- COLBS.- Estudio Epidemiológico Sobre Uso de Drogas en Lima. Rev. de Sanidad de los F2 policiales. Vol. 41: 1-8. Lima, 1980.

(24) JERI, FR. y COLBS.- Consumo de Drogas Peligrosas por Miembros de las Fuerzas Armadas y de la Fuerza Policial Peruana. Rev. Sanidad del Ministerio del Interior, 37: 104 - 112. Lima, 1976.

(25) JERI, F.R y COLABS.- El Síndrome de Pasta de Coca. Rev. Sanidad del Ministerio del Interior. 39: 1- 18, Lima, 1978. 
(26) JERI, F.R y COLABS.- Nuevos Observaciones Sobre los Síndromes Producidos por Fumar Pasta de Coca. En Cocaína 1980.: 86-95. Lima, 1980.

(27) JERI, R. - La Práctica de Fumar Pasta de Coca en Algunos Países de América Latina: Una Toxicomanía Grave y Generalizada Boletín de Estupefacientes XXXVI No. 2, abril - junio 1989.

(28) NIZAMA, M. - Síndrome de Pasta de Cocaína. Rev. Neuropsiquiatria T. XLII. Vols. 2 y $3-4 ; 114-$ 185, Lima, 1979.

(29) II Estudio de Consumo de Sustancias Psicoactivas en Colombia. Fundación Santafé de Bogotá. Dirección Nacional de Estupefacientes. 1996.

(30) VESPA UNIVERSITARIO. Sistema de Vigilancia Epidemiológica Sobre Consumo de Sustancias Psicoactivas en Población Universitaria. Alcaldía de Medellín. Secretaria de Salud. Red UNIR. Medellín. 1999.

(31) CEDRO: ROJAS, M. Epidemiología de Drogas en la Población Urbana Peruana. CEDRO. Area de Investigaciones, 1995. Lima.

(32) DOCUMENTO PROYECTO: Fortalecimiento de Estrategias de Reducción de la Demanda en Colombia, Ecuador y Venezuela. Equipo técnico Permanente: Dra Silvia Corella - CONSEP-; Lic. William Duque - MEC -; Lic. Paquita Moncayo MEC- ; Lic. Carlos Reyes - MEC -; Dr. Patricio Zapata - CONSEP -. Mimeo. Ecuador. 1999.

(33) ALCARAZ DEL CASTILLO, F.; SOLIZ V, R. M.; ZUAZO Y, J. El Uso Indebido de Drogas en Estudiantes de Bolivia, 1999. Estudio urbano, Rural. Centro Latinoaméricano de Investigación Científica. CELIN. Bolivia, 1999.

(34) PRIMER ESTUDIO NACIONAL SOBRE USO DE DROGAS, JUNIO DE 1999. Secretaria de Programación de la Drogadicción y la lucha contra el Narcotráfico. Presidencia de la Nación de la República de Argentina. Oficina de Prensa y Difusión. Re.pública de Argentina, 1999.

(35) SEGUNDO ESTUDIO NACIONAL SOBRE CONSUMO DE DROGAS EN CHILE, 1996. CONACE. Consejo Nacional para el Control de Estupefacientes. Sistema Nacional de Información sobre Drogas. Chile, 1996.

(36) BUENO R.; CARVALHO H,. Proyecto Brasil. Investigación sobre AIDS en usuarios de drogas en Brasil, Hucitec, San Pablo, 1999.
(37) RELATORIO ANUAL. Departamento de Investigaciones sobre Narcóticos- DENARC-. Sao Paulo. 1996.

(38) SIMONOVIS N.; PIRAS R.; DELGADO P, et al. Encuesta Domiciliaria sobre Salud y alcoholismo en la población del Municipio de Baruta. Miranda. CMDLT. En: Serie Investigaciones en Drogas No. 1. Investigaciones sobre el Consumo de Drogas en Venezuela. Resúmenes Analíticos. 2da Edición. Fundación Jose Felix Ribas. Centro de Documentación e Información- CEDIF -. Caracas, Venezuela, 1998. 78:26-27.

(39) AMESTY E., FUNDACIÓN JOSE FELIX RIBAS. Perfil del Usuario de Drogas. Trabajo Mimeografiado. En: Grupo Interinstitucional para la Investigación del Consumo de Drogas en Venezuela (Comp). Alcohol y otras Drogas en Venezuela. Caracas, 1995.

(40) SALAZAR M; RAMOS G,. Diagnóstico Epidemiológico del Consumo de Sustancias Psicoactivas en una Universidad Venezolana. Valencia, Universidad de Carabobo, 1994.

(41) SEPULVEDA G,. El Silencio de los Angustiados: Contextos Discursivos en el Consumo de Pasta Básica de Cocaína. En: Desintegración Social y Políticas Públicas en América Latina, Hopenhayn, M. Santiago de Chile. Naciones Unidas. Comisión Económica para América Latina y el Caribe, 1997.

(42) NAVARRO R; AREVALO M. Tratamiento y Rehabilitación de la Dependencia a la PBC, en: Pasta de Cocaína. Un Estudio Multidisciplinario. CEDRO, Perú 1984.

(43) NIZAMA, M. Sindrome de Pasta Básica de Cocaína. Rev. Neuropsiquiatria, 42:114,134. Perú 1979.

(44) OLIVER, E. Estrategias y Técnicas Terapéuticas en el Tratamiento de Adictos a la Pasta Básica de Cocaína. Revista Psicoactiva Vol. 2 No.1: 91 - 101, Perú, 1988.

(45) HURTADO j, . Institute Research of Drug. (Bolivia). Coca Interventions. Simposium Coca. IX Conferencia de Reducción de Daños. Sao Paulo. Brasil. 1998.

(46) LABIGALINI E,. Harm Reduction in the Use of Cannabis by Crack addicts in Brazil. IX Conferencia de Reducción de Daños. Sao Paulo. Brasil. 1998. 